index beat, with small bias and narrow limits of agreement [in brackets]: intra -1.1 [-4.9 to 2.7] and inter -0.7 [-5.4 to $3.9]$; verses 3 averaged beats $-1.1[-5.4$ to 3.1$]$ and -2 [-6.5 to 4.7]; 5 averaged beats -1.1 [-5.6 to 3.4$]$ and -1.2 [-6.6 to $4.2]$; and 10 averaged beats $-1.0[-5.0$ to 3.0$]$ and $-0.9[-6.2$ to 4.3]. Inter-observer limits of agreement for $\mathrm{E} / \mathrm{e}$ ' were also the narrowest for the index beat method: -1.6 [-0.4 to 3.7]; verses 3 averaged beats -0.6 [-3.5 to 2.2]; 5 averaged beats 0.4 [-3.1 to 2.2]; and 10 averaged beats -0.1 [-2.5 to 2.2].

Conclusion In patients with $\mathrm{AF}$, an index beat method is more reproducible than the conventional method of averaging multiple consecutive beats. This approach can enhance the reliability of measurements for both systolic and diastolic left ventricular function in patients with AF.

Conflict of Interest Nothing to declare

\section{THE IMPACT OF MISCLASSIFYING LEFT VENTRICULAR SIZE IF INDEXING TO BODY SURFACE AREA IS NOT PERFORMED}

${ }^{1}$ Carl Hayward, ${ }^{1}$ Carlos Perez ${ }^{*}$, ${ }^{2}$ Hitesh Patel, ${ }^{1}$ Kyriacos Mouyis, ${ }^{3}$ Ketna Patel, ${ }^{1}$ Mohammed Akhtar, 'Daniel Harding, 'Gamith Adasuriya, 'Holly Gillott, 'George Harvey, ${ }^{1}$ Imelda Sotto, 'Sanjeev Bhattacharyya. ${ }^{1}$ Barts Health NHS Trust; ${ }^{2}$ Imperial College; ${ }^{3}$ West Middlesex Hospital

\subsection{6/heartjnl-2019-BCS.11}

Introduction Guidelines recommend indexing measurements of left ventricle (LV) size to body surface area (BSA) to improve clinical validity. We sought to highlight the potential impact of misclassifying LV size in patients if indexing is not performed. Methods We reviewed the reports of all trans-thoracic echocardiograms performed at a large tertiary centre for cardiology and compared proportions of patients with LV dilatation based on LV internal diastolic dimension (LVIDd) $>5.8 \mathrm{~cm}$ (male), $>5.2 \mathrm{~cm}$ (female) or indexed (Dubois) LVIDd $>3.0 \mathrm{~cm} / \mathrm{m}^{2}$ (male), $>3.1 \mathrm{~cm} / \mathrm{m}^{2}$ (female). We also identified all reports with a diagnosis of moderate-severe or severe aortic regurgitation and compared proportions of patients with LV dilatation that would reach the threshold for surgical intervention when indexed and non-indexed values are used.

Results 20397 echocardiogram reports were reviewed. LV dilatation was present in $2821(13.8 \%)$ based on non-indexed LVIDd compared to $2083(10.2 \%)$ using indexed LVDD. After indexing for BSA 2202 (10.8\%) patients changed category: $1470(7.2 \%)$ patients deemed to have a dilated LV based on LVIDd were reclassified as normal, whereas 732 (3.6\%) patients deemed to have a normal sized LV were reclassified as dilated when LVIDd was indexed.

Reports of 71 patients with moderate-severe or severe aortic regurgitation were reviewed. $5(7.0 \%)$ had a LV internal systolic dimension (LVIDs) $>5 \mathrm{~cm}$ meeting criteria to consider surgery. When indexed to BSA, $15(21.1 \%)$ had indexed LVIDs $>2.5 \mathrm{~cm} / \mathrm{m}^{2}$ with $11(15.5 \%)$ changing from normal LVIDs to dilated LVIDs when indexed.

Conclusion Indexing left ventricular dimensions results in reclassification of $10.8 \%$ of patients, which could have implications on clinical management decisions. Furthermore, up to $15.5 \%$ of patients with aortic regurgitation may transition from non-dilated to dilated LVIDs when indexed values are used and so impact on timing of surgical referral. Uncorrected and BSA corrected quantitative measurements should be available on all echocardiogram reports.

Conflict of Interest None

\section{SEX DIFFERENCES IN THE AGREEMENT BETWEEN LEFT VENTRICULAR EJECTION FRACTION MEASURED BY MYOCARDIAL PERFUSION SCINTIGRAPHY AND BY ECHOCARDIOGRAPHY}

${ }^{1}$ Thang Han, ${ }^{2}$ Kishan Moosai*, ${ }^{2}$ Sams Jaker, ${ }^{2}$ Amjad Burgan, ${ }^{2}$ Adam Jacques, ${ }^{2}$ David Fluck, ${ }^{2}$ Mark MacGregor, ${ }^{2}$ Otar Lazariashvili, ${ }^{2}$ Vineet Prakash, ${ }^{2}$ Pankaj Sharma, ${ }^{2}$ Christopher Fry. ${ }^{1}$ Royal Holloway University of London; ${ }^{2}$ Ashford \& St Peter's NHS Foundation Trust

\subsection{6/heartjnl-2019-BCS.12}

Background Left ventricular ejection fraction (LVEF) is generally measured by echocardiography (Echo) but is increasingly available with myocardial perfusion scintigraphy (MPS). With MPS the threshold of LVEF below which there is a risk for myocardial infarct or sudden cardiac death is higher for women (51\%) than for men (43\%) [Shahir T, et al. J Nucl Cardiol 2006;13:495-506]. We tested the hypothesis that such a sex difference may also occur with Echo and MPS, by comparing LVEF measured by both methods.

Participants and setting: A total of 1141 patients underwent cardiac assessment, including resting LVEF measured both by MPS and Echo. Of these 743 patients (404 men, mean age $=67.7 \pm \mathrm{SD}=12.3 \mathrm{yr} ; 339$ women, $67.7 \pm 11.7 \mathrm{yr}$ ) had separate examinations within 6months and their data used for analysis. A subset of 327 of these patients (181 men, 68.8 $\pm 12.1 \mathrm{yr} ; 146$ women, $66.4 \pm 12.1 \mathrm{yr}$ ) had examinations at a shorter time apart (within 1month) and were additionally analysed as this sub-group. All examinations were at Ashford \& St Peter's NHS Foundation Trust between 30-11-2012 and 30-05-2017 (figure 1).

Methods The rest MPS was performed with the injection of 99mTc-tetrofosmin (600-1000MBq). Images were obtained using dual-head SPECT cameras (Siemens Symbia S, Erlangen, Germany) and LVEF was determined from gated images. The rest Echo was performed with a high-end 2-dimensional echocardiographic unit (Sonos 5500, Andover, Mass., US or Vingmed System V, Horten, Norway) and images were acquired with standard parasternal, short-axis and apical views and LVEF was calculated by the modified Simpson's biplane disks method. Agreement between MPS and Echo (neither considered as a reference method) was assessed by Bland-Altman plots: LVEF difference (MPS minus Echo) against average LVEF ((MPS+Echo)/2). Data are presented as means and $\pm \mathrm{SD}$. Results Of patients who had MPS and Echo examinations within 6months, mean LVEF difference was $+1.1 \%$ (95\% limits

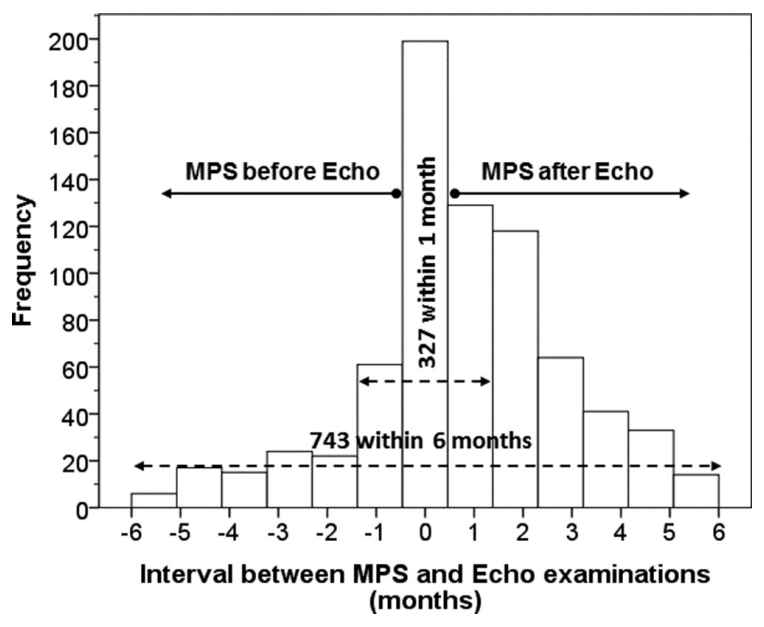

Abstract 12 Figure 1 